\title{
Discrimination of basal cell carcinoma from normal dermal stroma by quantitative multiphoton imaging
}

\author{
Sung-Jan Lin* and Shiou-Hwa Jee* \\ Department of Dermatology, National Taiwan University Hospital and National Taiwan University College of \\ Medicine, Taipei 100, Taiwan \\ Chien-Jui Kuo and Ruei-Jr Wu \\ Department of Physics, National Taiwan University, Taipei 100, Taiwan \\ Wei-Chou Lin \\ Department of Pathology, National Taiwan University Hospital, Taipei 106, Taiwan \\ Jau-Shiuh Chen, Yi-Hua Liao, Chih-Jung Hsu, and Tsen-Fang Tsai \\ Department of Dermatology, National Taiwan University Hospital and National Taiwan University College of \\ Medicine, Taipei 100, Taiwan \\ Yang-Fang Chen and Chen-Yuan Dong \\ Department of Physics, National Taiwan University, Taipei 106, Taiwan
}

Received April 20, 2006; revised June 24, 2006; accepted July 3, 2006; posted July 10, 2006 (Doc. ID 70148); published August 25, 2006

\begin{abstract}
We performed multiphoton fluorescence (MF) and second-harmonic generation (SHG) imaging on human basal cell carcinoma samples. In the dermis, basal cell carcinomas can be identified by masses of autofluorescent cells with relatively large nuclei and marked peripheral palisading. In the normal dermis, SHG from dermal collagen contributes largely to the multiphoton signal. However, within the cancer stroma, SHG signals diminish and are replaced by autofluorescent signals, indicating that normal collagen structures responsible for SHG have been altered. To better delineate the cancer cells and cancer stroma from the normal dermis, a quantitative MF to SHG index is developed. We demonstrate that this index can be used to differentiate cancer cells and adjacent cancer stroma from the normal dermis. Our work shows that MF and SHG imaging can be an alternative for Mohs' surgery in the real-time guidance of the secure removal of basal cell carcinoma. (C) 2006 Optical Society of America
\end{abstract}

OCIS codes: $190.4160,170.3880,180.0180$.

Basal cell carcinoma is the most prevalent skin cancer and accounts for 800,000 cases per year in the United States, ${ }^{1}$ with an annual incidence rate of nearly 200 for every 100,000 women and 400 for every 100,000 men. ${ }^{1}$ Excessive sunlight exposure is an important risk factor for this disease, and the tumor most often develops on the head and neck areas. Though rarely metastasizing, basal cell carcinoma may lead to local tissue destruction. Usually surgical removal is recommended for patients with this tumor. However, the margin of cancer cannot be securely determined during operation, and the local recurrence rate is higher than $10 \%$ in cases undergoing simple excision. For secure removal of the entire cancer tissue, the technique of Mohs' micrographic surgery is used. This technique can detect cancer margin with approximately $100 \%$ certainty, and the recurrence rates are only $1 \% .{ }^{2}$ However, Mohs' micrographic surgery is time consuming. Further, it requires the simultaneous cooperation of the technician for specimen processing and the surgeon. The development of a real-time imaging method for the margin determination of basal cell carcinoma capable of circumventing the above-mentioned drawbacks will be invaluable for dermatological applications.

The nonlinear optical technique of multiphoton fluorescence (MF) imaging, employing an ultrafast laser for effective excitation of fluorescent materials, has been used for biological specimen imaging both in vivo and in vitro. ${ }^{3-7}$ Due to the nonlinear excitation process, only the focal volume can be effectively excited by use of a near-infrared light source, allowing higher axial contrast, increased imaging depth, and lower overall sample photodamage to be achieved. Moreover, the easy access to the emitted fluorescence also enables further analysis of the spectral fingerprinting as well as other characteristic features, such as fluorescence lifetime, to be determined. ${ }^{3,8}$ In addition, the nonlinear optical effect of second-harmonic generation (SHG) has also been widely used in biomedical imaging. ${ }^{8-10}$ In brief, the interaction of the incident laser with noncentrosymmetric biological structures, such as collagen and muscle fibers, leads to the generation of photons 
at a wavelength of exactly half the wavelength of the incident light. ${ }^{8-10}$ Unlike fluorescence excitation, no molecular transition is involved in the SHG process. Therefore, sample photodamage is minimal. Because SHG is structurally sensitive, transitions of biomolecular structures can be monitored. ${ }^{8-13}$ These two techniques, which are minimally destructive and do not rely on the use of labeling dyes and pretreatments of samples, are highly suitable for in vivo application. In this ex vivo study, we combined MF and SHG imaging to investigate the feasibility of multiphoton imaging in identifying the margin of human basal cell carcinoma.

The MF and SHG microscopic system used in this study is a modified version of a homebuilt laser scanning microscopic imaging system based on an upright microscope (E800, Nikon, Japan) described previously. ${ }^{4}$ The $760 \mathrm{~nm}$ output of the Ti:sapphire laser is scanned in the focal plane by a galvanometerdriver $x-y$ mirror scanning system (Model 6220, Cambridge Technology, Cambridge, Mass.). A shortpass dichroic mirror (700DCSPRUV, Chroma Technology, Brattleboro, Vt.) is used to reflect the incident excitation laser source to the sample. The average laser power at the sample is $4.5 \mathrm{~mW}$, and an oilimmersion objective (S Fluor $40 \times$, NA 1.3, Nikon) is used for high-resolution imaging. The generated MF and SHG signals are collected by the same objective. Prior to reaching the photodetectors, the MF and SHG signals are separated by a secondary dichroic mirror (435DCSX, Chroma Technology). The SHG signal centered at $380 \mathrm{~nm}$ is reflected by the secondary dichroic and further filtered by a bandpass filter (HQ380/20, Chroma Technology), while the longer wavelength MF passes through the dichroic mirror and a broadband pass filter (E435LP, Chroma Technology) before being detected. The detection bandwidths of the SHG and MF signals are $370-390 \mathrm{~nm}$ and $435-700 \mathrm{~nm}$, respectively. The signal photons are processed by a single-photon counting photomultiplier tube (R7400P, Hamamatsu, Japan) and a homebuilt discriminator.

Nine nodular type basal cell carcinoma specimens are imaged in this study. The study protocol was approved by our Institutional Review Board. We conformed to the Helsinki Declaration with respect to human subjects in biomedical research. For better comparison of MF/SHG images of the cells and tissue with histological results, we used formalin-fixed specimens for analysis. Serial thin cross-section slices (7-10 $\mu \mathrm{m}$ in thickness) of each specimen were used for multiphoton imaging and histological examinations. A thin cross-section slice of each specimen was mounted on the slide and covered with a No. 1.5 coverslip for viewing. Large area multiphoton imaging was performed for each specimen. For quantitative analysis of the specimens, we randomly selected five $50 \mu \mathrm{m} \times 50 \mu \mathrm{m}$ rectangular areas inside the cancer masses, cancer stroma, and normal stroma in the reticular dermis, respectively, in each sample for quantitative analysis. Cancer stroma includes stroma within the cancer clumps and right adjacent to the cancer mass. Normal stroma is defined as the

dermal stroma at least $200 \mu \mathrm{m}$ away from the margin of the tumor clumps. Because adequate normal dermal stroma and cancer stroma were not present in all nine specimens, three representative specimens containing cancer clumps, cancer stroma, and normal dermis were used for quantitative analysis. In each selected area, the pixels of MF are defined as $a$ and pixels of SHG are defined as $b$. The MF to SHG index (MFSI) is defined as $(a-b) /(a+b)$. In this nomenclature, MFSI approaches the maximum value of 1 when only MF signals are present. The smallest value of MFSI is -1 , and this occurs when MF is absent and only the SHG signal is present. The index of each selected area is computed and the average index in each case is calculated. For comparison, the adjacent sections of each specimen were further processed for histological examination with hematoxylin and eosin (H\&E) stains and elastic stains (Verhoeffvan Gieson stain).

Figure 1 shows the MF/SHG and histological images of a representative specimen, and the quantitative analysis of MFSI is shown in Fig. 2. Several interesting results are obtained. First, in the normal dermal stroma (Fig. 1), the SHG accounts for most of the signals, and the autofluorescent elastic fibers are found to intersperse within the fibrous collagen matrix (data of elastic stain not shown). This reflects the fact that collagen is the major extracellular matrix protein in the dermis. Since normal collagen is ineffective in producing MF signals when a longer wavelength is used for multiphoton imaging, ${ }^{4,8}$ SHG signals can be used to analyze the dermal fibrous structures of collagen. Within the tumor regions, aggregates of autofluorescent cancer cells can be visualized [Fig. 1(a)]. In the magnified image [Fig. 1(a), yellow inset], cells with relatively large nuclei can be found and the peripheral cells in each cancer clump are palisading along the basement membrane, a

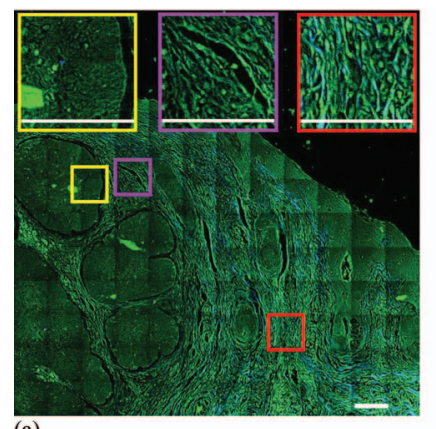

(a)

Fig. 1. (a) MF/SHG image and (b) H\&E stain. Yellow insets, cancer clump; purple insets, cancer stroma; red insets, normal dermis. Basal cell carcinoma is featured by clumps of autofluorescent cells with relatively large nuclei in the dermis (a). The autofluorescent peripheral cells in each cancer clump are palisading along the basement membrane [(a), yellow inset]. The result is consistent with the histology [(b), yellow inset]. The cancer stroma has decreased SHG signals [(a), purple inset] as compared with that of normal dermal stroma [(a), red inset]. The change in collagen content is better appreciated in MF/SHG image than that of routine histology [(b), purple and red insets]. [SHG signals are blue and MF signals are green in (a). Bars, $110 \mu \mathrm{m}$. 


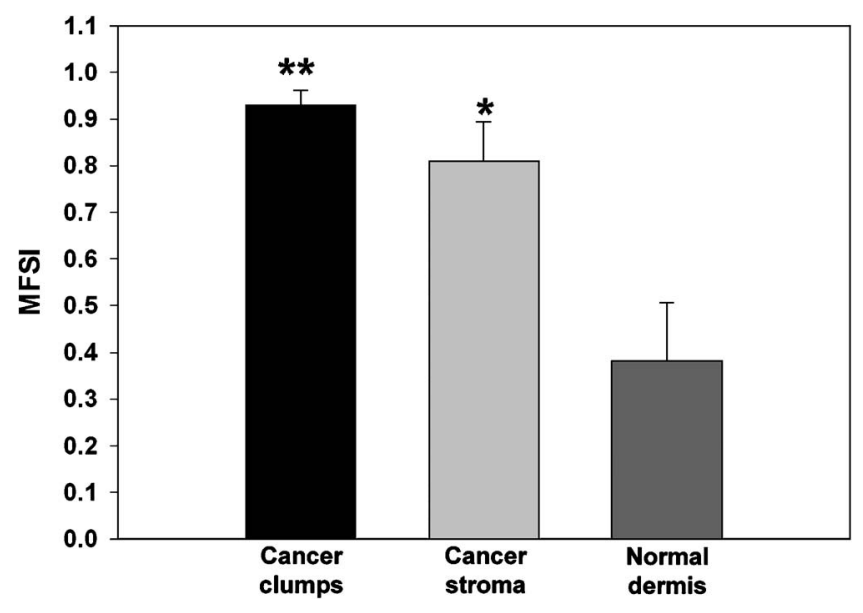

Fig. 2. MFSI of basal cell carcinoma clumps, cancer stroma, and normal dermal stroma. The MFSI within cancer clumps and cancer stroma are significantly greater than that of normal dermal stroma. Error bars indicate calculated standard deviations. A student t-test is performed against normal dermis for comparison. ${ }^{*} p<0.01$; ${ }^{* *} p$ $<0.0001$.

unique feature of basal cell carcinoma. This is consistent with the histological results [Fig. 1(b)]. In the cancer stroma, the SHG signals are greatly diminished and MF signals increase [Fig. 1(a), purple inset] in comparison with that of normal stroma [Fig. 1(a), red inset]. Since the SHG signals of collagen depend on the regular packing of triple-helical collagen molecules, the diminished SHG signals in the cancer stroma indicate the fact that the collagen structures responsible for SHG are disrupted or the collagen molecules themselves are deficient. Our result is consistent with a previous report that collagen content in the basal cell carcinoma stroma is decreased as compared with normal dermal stroma. ${ }^{14}$ It has also been shown that matrix metalloproteinase expression is upregulated in basal cell carcinoma, ${ }^{15}$ and the collagenolytic activity may lead to the disruption of collagen in the adjacent stroma. Elastic fiber stains reveal no increased elastic fibers in the cancer stroma (data not shown). The origin of the autofluorescent signals in the cancer stroma is unclear at this stage.

To better quantify the changes of MF and SHG in the cancer, the MFSI is shown in Fig. 2. The MFSI is highest within the tumor masses (mean MFSI =0.93) where the contribution of the fluorescent signal comes from the cytoplasm. In the normal dermal stroma, the MFSI is the lowest, indicating the relatively high content of intact collagen molecules. In the cancer stroma, the MFSI is significantly higher than that of normal dermal stroma. The decrease of SHG and increase of MF in cancer stroma accounts for the higher MFSI in comparison with that of normal dermal stroma. When in doubt, this higher MFSI in cancer stroma may help to delineate the cancer area from normal dermis during the surgical removal of basal cell carcinoma.

In conclusion, our work shows that discrimination of basal cell carcinomas from the normal dermis can be achieved by multiphoton imaging. Basal cell carcinomas are revealed to be clumps of autofluorescent cells with relatively large nuclei and marked peripheral palisading. Compared with the normal dermis, the SHG signals decrease and MF signals increase in the stroma within and adjacent to the tumor masses. This may reflect an upregulated collagenolytic activity of basal cell carcinoma. Quantitative analysis by use of the MFSI values can help to differentiate cancer cells and cancer stroma from normal dermal stroma. Our results show that MF and SHG imaging can serve as an alternative for Mohs' surgery in the guidance of the surgical removal of basal cell carcinomas.

C.-Y. Dong's e-mail address is cydong@phys.ntu.edu.tw.

*These authors contributed equally to this work.

\section{References}

1. A. I. Rubin, E. H. Chen, and D. Ratner, N. Engl. J. Med. 353, 2262 (2005).

2. D. E. Rowe, R. J. Carroll, and C. L. Day, Jr., J. Dermatol. Surg. Oncol. 15, 315 (1989).

3. K. Konig and I. Riemann, J. Biomed. Opt. 8, 432 (2003).

4. S. J. Lin, R. J. Wu, H. Y. Tan, W. Lo, W. C. Lin, T. H. Young, C. J. Hsu, J. S. Chen, S. H. Jee, and C.-Y. Dong, Opt. Lett. 30, 2275 (2005).

5. S. W. Teng, H. Y. Tan, J. L. Peng, H. H. Lin, K. H. Kim, W. Lo, Y. Sun, W. C. Lin, S. J. Lin, S. H. Jee, P. T. C. So, and C.-Y. Dong, Invest. Ophthalmol. Visual Sci. 47, 1216 (2006).

6. Y. Sun, J. Su, W. Lo, S. J. Lin, S. H. Jee, and C.-Y. Dong, Opt. Express 11, 3377 (2003).

7. W. Zipfel, R. Williams, R. Christie, A. Nikitin, B. Hyman, and W. Webb, Proc. Natl. Acad. Sci. U.S.A. 100, 7075 (2003).

8. A. Zoumi, A. Yeh, and B. J. Tromberg, Proc. Natl. Acad. Sci. U.S.A. 99, 11014 (2002).

9. E. Brown, T. McKee, E. diTomaso, A. Pluen, B. Seed, Y. Boucher, and R. K. Jain, Nat. Med. 9, 796 (2003).

10. P. J. Campagnola and L. M. Loew, Nat. Biotechnol. 21, 1356 (2003).

11. S. J. Lin, C. Y. Hsiao, Y. Sun, W. Lo, W. C. Lin, G. J. Jan, S. H. Jee, and C.-Y. Dong, Opt. Lett. 30, 622 (2005).

12. H. Y. Tan, S. W. Teng, W. Lo, W. C. Lin, S. J. Lin, S. H. Jee, and C.-Y. Dong, J. Biomed. Opt. 10, 54019 (2005).

13. S. J. Lin, W. Lo, H. Y. Tan, J. Y. Chan, W. L. Chen, S. H. Wang, Y. Sun, W. C. Lin, J. S. Chen, C. J. Hsu, J. W. Tjiu, H. S. Yu, S. H. Jee, and C.-Y. Dong, J. Biomed. Opt. 11, 034020 (2006).

14. A. Nijssen, T. C. Bakker Schut, F. Heule, P. J. Caspers, D. P. Hayes, M. H. A. Neumann, and G. J. Puppels, J. Invest. Dermatol. 119, 64 (2002).

15. T. Yucel, A. Mutnal, K. Fay, S. E. Fligiel, T. Wang, T. Johnson, S. R. Baker, and J. Varani, Exp. Mol. Pathol. 79, 151 (2005). 